\title{
Morita Equivalence of Poisson Manifolds
}

\author{
Ping $\mathbf{X u}^{\star}$ \\ Department of Mathematics, University of Pennsylvania, Philadelphia, PA 19104, USA
}

Received November 19, 1990; in revised form April 12, 1991

\begin{abstract}
Poisson manifolds are the classical analogue of associative algebras. For Poisson manifolds, symplectic realizations play a similar role as representations do for associative algebras. In this paper, the notion of Morita equivalence of Poisson manifolds, a classical analogue of Morita equivalence of algebras, is introduced and studied. It is proved that Morita equivalent Poisson manifolds have equivalent "categories" of complete symplectic realizations. For certain types of Poisson manifolds, the geometric invariants of Morita equivalence are also investigated.
\end{abstract}

\section{Introduction}

Poisson manifolds are the classical analogue of $C^{*}$-algebras (or noncommutative algebras). One can find counterparts in Poisson geometry for many concepts in $C^{*}$-algebras. For instance, representations of $C^{*}$-algebras correspond to symplectic realizations of Poisson manifolds, traces of $C^{*}$-algebras to invariant measures on Poisson manifolds, automorphism groups of $C^{*}$-algebras to Poisson flows on Poisson manifolds, etc. The similarities between these two distinct subjects are more than conceptual; they are also reflected in the techniques and methods of studying these subjects. Therefore, it would be useful in the study of Poisson geometry to develop comparable techniques to those used in the theory of $C^{*}$-algebras, such as the theory of Morita equivalence.

The theory of Morita equivalence goes back to Morita [Mo] in the 1950's, who proved the fundamental theorem: two rings have equivalent categories of left modules if and only if there exists an equivalence bimodule for the rings. This concept of equivalence was first generalized to the context of $C^{*}$-algebras, in the name of strong Morita equivalence, by Marc Rieffel [Rie1-Rie4]. It turns out now to be one of the most important equivalence relations in $C^{*}$-algebras, playing a crucial role in understanding the structures of some $C^{*}$-algebras, such as

\footnotetext{
* (ping@ math.upenn.edu)
} 
transformation $C^{*}$-algebras and foliation $C^{*}$-algebras. Morita equivalent $C^{*}$-algebras have many similar features. For instance, they have equivalent categories of Hermitian left modules, isomorphic $K$-groups, and so on.

In the present paper, we introduce and study an equivalence relation for integrable Poisson manifolds, which we expect to play a similar role for Poisson manifolds that Morita equivalence plays for $C^{*}$-algebras. As we know, the role of symplectic realizations in the theory of Poisson manifolds is similar to that of representations in the study of noncommutative algebras. One of the main theorems in this work is that Morita equivalent Poisson manifolds have equivalent "categories" of complete symplectic realizations, a result parallel to the fundamental theorem in the theory of Morita equivalence of $C^{*}$-algebras. In fact, this machinery of Morita equivalence, it turns out, is very successful in handling the computation of realizations for some particularly interesting Poisson manifolds [X2].

The notion of Morita equivalence of symplectic groupoids introduced in [X1] plays an essential role in this work. In fact, we show that the $\alpha$-simply connected symplectic groupoids of Morita equivalent Poisson manifolds are Morita equivalent as symplectic groupoids.

In Poisson geometry, a large variety of interesting Poisson manifolds are regular Poisson manifolds; therefore Morita equivalence for regular Poisson manifolds is of particular interest to us. For symplectic manifolds, a complete invariant of Morita equivalence is just the fundamental group. In general, however, it is far from completely solved as to what quantities of Poisson manifolds completely classify the Morita equivalence classes of Poisson manifolds. So far, two interesting results have been obtained in this direction. One is that a regular Poisson manifold is Morita equivalent to a zero Poisson manifold if and only if its symplectic leaves are all simply connected and its fundamental class vanishes, or equivalently, if and only if the regular Poisson manifold is a locally trivial bundle of simply connected symplectic manifolds. The other is that for Poisson manifolds in which the characteristic foliations are trivial fibrations $\pi: P=S \times Q \rightarrow Q$, the variation lattice $\mathscr{R}^{Q}$ of symplectic structures along symplectic leaves is a complete Morita equivalence invariant. These facts suggest that, roughly speaking, Morita equivalence is an equivalence that measures the symplectic leaf space and the variation of the symplectic structures along symplectic leaves of a Poisson manifold.

This paper is organized as follows.

Some basic facts concerning Morita equivalence of symplectic groupoids are recalled in Sect. 1. In Sect. 2, the definition of Morita equivalence of Poisson manifolds is introduced and several interesting examples are discussed. Section 3 is devoted to the discussion of symplectic realizations of Morita equivalent Poisson manifolds, and Sect. 4 and 5 are to the Morita equivalence of regular Poisson manifolds as described above.

Finally, note that in this paper, manifold always means a connected smooth manifolds, and symplectic groupoids are always assumed to be $\alpha$-connected.

\section{Morita Equivalence of Symplectic Groupoids}

The notion of Morita equivalence of symplectic groupoids was introduced in [X1], based on the notion of equivalent topological groupoids introduced in [MRW]. In this section, we recall some basic facts concerning this Morita equivalence. 
Definition 1.1. Symplectic groupoids $G \rightrightarrows G_{0}$ and $H \rightrightarrows H_{0}$ are called Morita equivalent if there exists a symplectic manifold $X$ and surjective submersions $\rho$ from $X$ to $G_{0}$ and $\sigma$ from $X$ to $H_{0}$ such that

1. $G$ has a free and proper left action on $X$;

2. $H$ has a free and proper right action on $X$;

3. the two actions commute with each other;

4. $\rho$ induces a diffeomorphism from the quotient space $X / H$ onto $G_{0}$;

5. $\sigma$ induces a diffeomorphism from the quotient space $G \backslash X$ onto $H_{0}$; and

6. the graph of the $G, H$ actions:

$$
\begin{aligned}
\Omega & =\{(g, x, h, g \cdot x \cdot h) \mid g \in G, h \in H \text { and } x \in X \text { such that } g \cdot x \cdot h \text { makes sense }\} \\
& \subset G \times X \times H \times X^{-}
\end{aligned}
$$

is a lagrangian submanifold, where $X^{-}$denotes the symplectic manifold $X$ with the opposite symplectic structure.

$(X ; \rho ; \sigma)$ is called an equivalence bimodule between symplectic groupoids $G$ and $H$.

It is known that this Morita equivalence indeed induces an equivalence relation among symplectic groupoids [X1].

Given a symplectic groupoid $G \rightrightarrows G_{0}$, by a symplectic left $G$-module, we mean a symplectic manifold $F$ together with a smooth map $J: F \rightarrow G_{0}$ such that $F \stackrel{J}{\rightarrow} G_{0}$ admits a symplectic left $G$-action. $J$ is usually called the momentum mapping of the module $F$. The symplectic left modules of a given symplectic groupoid $G$ becomes a "category" $\zeta(G)$ in the following sense: the objects of $\zeta(G)$ are symplectic left $G$-modules, the morphisms are canonical relations satisfying certain compatible conditions with the groupoid actions, and the composition of morphisms is set-theoretic composition of relations. More precisely, a morphism from a symplectic left $G$-module $F_{1}$ to a symplectic left $G$-module $F_{2}$ is a lagrangian submanifold $\mathscr{L} \subset F_{2} *_{G_{0}} F_{1}^{-}$that is invariant under the diagonal action of $G$. In fact, $\zeta(G)$ is not a true category. However, it does satisfy all the axioms of a usual category except that it requires the transversality assumption for the composition of morphisms being a morphism. A fundamental result regarding Morita equivalence of symplectic groupoids is the following:

Theorem 1.1. Morita equivalent symplectic groupoids have equivalent "categories" of symplectic left modules.

\section{Morita Equivalence of Poisson Manifolds}

With the preliminary in Sect. 1, we can introduce the concept of Morita equivalence for Poisson manifolds.

Definition 2.1. Two Poisson manifolds $P_{1}$ and $P_{2}$ are said to be Morita equivalent if there exists a symplectic manifold $X$ together with a Poisson morphism $\rho: X \rightarrow P_{1}$ and an anti-Poisson morphism $\sigma: X \rightarrow P_{2}$ such that $P_{1} \stackrel{\rho}{\leftarrow} X \stackrel{\sigma}{\rightarrow} P_{2}$ is a complete full dual pair [W1] with connected and simply connected fibres. $X$ is called an equivalence bimodule. 
We say that a dual pair $P_{1} \stackrel{\rho}{\leftarrow} X \stackrel{\sigma}{\rightarrow} P_{2}$ is complete if both $\rho$ and $\sigma$ are complete in the sense that the pull back of any complete Hamiltonian is still a complete Hamiltonian on $X$.

Remark. (1) The idea to relate Poisson manifolds by means of dual pairs is due to Weinstein. These dual pairs were studied in [W1], where many similarities between related Poisson manifolds were discovered.

(2) It was proved by Weinstein that such a dual pair as in the preceding definition naturally induces an affinoid structure on $X$ [W3]. Consequently, both $P_{1}$ and $P_{2}$ are automatically integrable in the sense of Dazord [D1]. Namely, they admit global symplectic groupoids. In fact, the horizontal and vertical groupoids arising from the symplectic affinoid structure on $X$ are exactly the symplectic groupoids over $P_{1}$ and $P_{2}$, respectively.

Many examples of Morita equivalent Poisson manifolds arise from Poisson reduction theory, to be discussed in [X2]. Here, we list some interesting simple examples.

Example 2.1. Let $\mathrm{S}$ be a connected and simply connected symplectic manifold, $M$ a connected manifold with zero Poisson structure; then the direct product $S \times M$ is Morita equivalent to $M$. In fact, if we take $X=S \times T^{*} M, \rho=(\mathrm{id}, \mathrm{pr}): X \rightarrow S \times M$, and $\sigma=\operatorname{pr}: X \rightarrow M$, where pr is the natural projection from $T^{*} M$ onto $M$, then $S \times M \leftarrow X \rightarrow M$ is a Morita equivalence as defined above.

In particular, every connected and simply connected symplectic manifold is Morita equivalent to a one point space with zero Poission structure. Therefore, simply connected symplectic manifolds play a similar role in Poisson geometry as the algebra of compact operators in $C^{*}$-algebras.

Example 2.2. Let $(S, \omega)$ be any symplectic manifold with symplectic form $\omega$; then $(S, a \omega)$ is Morita equivalent to $(S, b \omega)$, provided that $a b \neq 0$.

In order to see this, we take $X$ to be the fundamental groupoid $\Pi_{1}(S)$, equipped with the symplectic structure $\tilde{\omega}=a \rho^{*} \omega-b \sigma^{*} \omega$, where $\rho$ and $\sigma$ are the source and target maps of the groupoid $\Pi_{1}(S)$,respectively. It is clear that $\rho: \Pi_{1}(S) \rightarrow(S, a \omega)$ is a Poisson morphism, while $\sigma: \Pi_{1}(S) \rightarrow(S, b \omega)$ is anti-Poisson. For any $v_{1}, v_{2} \in T_{x} X$ such that $v_{1}$ is tangent to the $\rho$-fibre through $x$ and $v_{2}$ is tangent to the $\sigma$-fibre through $x$,

$$
\begin{aligned}
\tilde{\omega}\left(v_{1}, v_{2}\right) & =\left(a \rho^{*} \omega\right)\left(v_{1}, v_{2}\right)-\left(b \sigma^{*} \omega\right)\left(v_{1}, v_{2}\right) \\
& =a \omega\left(T \rho v_{1}, T \rho v_{2}\right)-b \omega\left(T \sigma v_{1}, T \sigma v_{2}\right) \\
& =0 .
\end{aligned}
$$

I.e., $\rho$-fibres and $\sigma$-fibres are $\tilde{\omega}$-orthogonal. Therefore, it follows immediately that $(S, a \omega)$ and $(S, b \omega)$ are Morita equivalent as Poisson manifolds.

In fact, Weinstein has observed that for symplectic manifolds a complete invariant of Morita equivalence is just the fundamental group.

Proposition 2.1. Let $S_{1}$ and $S_{2}$ be any symplectic manifolds. $S_{1}$ is Morita equivalent to $S_{2}$ if and only if $\pi_{1}\left(S_{1}\right) \cong \pi_{1}\left(S_{2}\right)$.

Proof. Suppose that $S_{1}$ and $S_{2}$ are Morita equivalent with equivalence bimodule $(X ; \rho ; \sigma)$. Then $\rho: X \rightarrow S_{1}$ is a fibration with connected and simply connected fibres. 
It follows from the exact sequence

$$
0=\pi_{1}(\rho-\text { fibre }) \rightarrow \pi_{1}(X) \rightarrow \pi_{1}\left(S_{1}\right) \rightarrow \pi_{0}(\rho \text {-fibre })=0
$$

that $\pi_{1}(X) \cong \pi_{1}\left(S_{1}\right)$. Similarly, $\pi_{1}(X) \cong \pi_{1}\left(S_{2}\right)$. Hence, $\pi_{1}\left(S_{1}\right) \cong \pi_{1}\left(S_{2}\right)$.

Conversely, let $\widetilde{S}_{1}$ and $\widetilde{S}_{1}$ be the universal covering spaces of $S_{1}$ and $S_{2}$, respectively, and $G=\pi_{1}\left(S_{1}\right) \cong \pi_{1}\left(S_{2}\right)$. Take $X=\left(\tilde{S}_{1} \times \widetilde{S}_{2}\right) / G$ equipped with an obvious symplectic structure obtained in a similar way as in Example 2.2, where $G$ acts on $\tilde{S}_{1} \times \tilde{S}_{2}$ diagonally. Let $\rho: X \rightarrow S_{1}$ and $\sigma: X \rightarrow S_{2}$ be the two natural projections. It is clear that $S_{1} \stackrel{\rho}{\leftarrow} X \stackrel{\sigma}{\rightarrow} S_{2}$ is an equivalence bimodule. Q.E.D.

Remark. For any given fundamental group (i.e., a group which is a fundamental group of a certain manifold), there always exists a symplectic manifold with this group as its fundamental group (one can simply take the contangent bundle of the manfold having this fundamental group as the symplectic manifold). Therefore, the Morita equivalent classes of symplectic manifolds are in one-to-one correspondence with the fundamental groups of manifolds.

\section{Symplectic Realizations of Morita Equivalent Poisson Manifolds}

For Poisson manifolds, symplectic realizations play a similar role as representations do for $C^{*}$-algebras. Therefore, the study of symplectic realizations of Poisson manifolds is of particular importance in Poisson geometry. First of all, let us recall the definition of symplectic realizations.

Definition 3.1. ([CDW]) Let $P$ be a Poisson manifold. $A$ symplectic realization of $P$ is a pair $(X, \rho)$, where $X$ is a symplectic manifold and $\rho$ is a Poisson morphism from $X$ to $P$. A symplectic realization $\rho: X \rightarrow P$ is called complete if $\rho$ is complete as a Poisson map, i.e., the pull back of every compactly supported function on $\rho$ has a complete Hamiltonian vector field on $X$; a symplectic realization is said to be full if it is a submersion.

Suppose that $(\Gamma \rightrightarrows P, \alpha, \beta)$ is a symplectic groupoid and $\rho: X \rightarrow P$ is a symplectic realization of $P$. According to Theorem 1.1 in Chap. 3 of [CDW], $\Gamma$ has a local action on $X$. In general, it is not a global action. However, if $\Gamma$ is $\alpha$-simply connected, a remarkable fact concerning complete symplectic realizations emerges that any complete symplectic realization of $P$ becomes a symplectic left $\Gamma$-module in a natural way. In other words, $\Gamma$ has a global action on any complete symplectic realization of $P$. This fact has also been proved independently by Dazord [D1].

Theorem 3.1. Let $\Gamma$ be a symplectic groupoid over $P$. If $X$ is a symplectic left $\Gamma$-module, then its momentum mapping $\rho: X \rightarrow P$ is a complete symplectic realization. Conversely, if $\Gamma$ is $\alpha$-simply connected and $\rho: X \rightarrow P$ is a complete symplectic realization, then $X$ naturally becomes a symplectic left $\Gamma$-module.

Proof. Suppose that $\rho: X \rightarrow P$ is the momentum mapping of a symplectic left $\Gamma$-module; then $\rho$ is a symplectic realization [MiW]. Given a complete Hamiltonian $f \in C^{\infty}(P)$, we denote the Hamiltonian flow of $\alpha^{*} f$ on $\Gamma$ and the Hamiltonian flow of $\rho^{*} f$ on $X$ by $\phi_{t}^{\alpha}$ and $\phi_{t}^{\rho}$, respectively. Then it is clear that for any given $x \in X$, 
$\phi_{t}^{\rho}(x)=\left(\phi_{t}^{\alpha} u\right) \cdot x$, where $u=\rho(x)$ [CDW]. Since $\alpha$ is always complete as a source map of the symplectic groupoid $\Gamma$, therefore $\phi_{t}^{\rho}$ is also complete.

Conversely, according to Theorem 1.1 in Chap. 3 of [CDW], there exists a lagrangian immersion $\Lambda \subset \Gamma \times X \times X^{-}$, maximal among all lagrangian immersions contained in $K=\{(r, x, y) \mid \rho(y)=\alpha(r)\}$ and containing $I=\{(u, x, x) \mid \rho(x)=u\}$ as a submanifold. It is clear that $(r, x, y) \in \Lambda$ if and only if there exists a point $u \in P$ and $k$ functions $h_{i} \in C^{\infty}(P)$ such that if $\phi^{\alpha}$ and $\phi^{\rho}$ denote the products of Hamiltonian flows of $\alpha^{*} h_{i}$ and $\rho^{*} h_{i}$ in the same order, respectively, $r=\phi^{\alpha}(u)$ and $y=\phi^{\rho}(x)$. It is easy to see that $\beta(r)=\rho(x)=u$.

By $p$, we denote the projection from $\Gamma \times X \times X^{-}$onto $\Gamma \times X$ given by $p(r, x, y)=(r, x)$. First of all, we claim that $p(\Lambda)=\Gamma_{* P} X=\{(r, x) \mid \beta(r)=\rho(x)\}$. To prove this, it suffices to show that $\Gamma *_{P} X \subseteq p(\Lambda)$. Given any $(r, x) \in \Gamma *_{P} X$, we assume that $u=\beta(r)=\rho(x)$. Since $\beta$-fibres are connected, there exists a product of Hamiltonian flows $\phi^{\alpha}$ generated by $\alpha^{*} h_{i}$, such that $r=\phi^{\alpha} u$. We denote by $\phi^{\rho}$ the corresponding product of Hamiltonian flows generated by $\rho^{*} h_{i}$ in the same order. It follows from the completeness of $\rho$ that $\phi^{\rho}$ always exists. Let $y=\phi^{\rho}(x)$; then it is clear that $(r, x, y) \in \Lambda$; therefore, $(r, x) \in p(\Lambda)$.

Moreover, $\Lambda$ is a graph over $\Gamma *_{P} X$.

Let $p_{i}$ denote the natural projection from $\Lambda$ onto its $i^{\text {th }}$ factor $(i=1,2,3)$. For any $x \in X$, let $u=\rho(x) \in P$. Consider the map $p_{1}: p_{2}^{-1}(x) \rightarrow \beta^{-1}(u)$. Since $p_{2}: \Lambda \rightarrow X$ is a submersion, $\operatorname{dim} p_{2}^{-1}(x)=\operatorname{dim} \beta^{-1}(u)$ by dimension counting. In fact, $p_{1}$ has the path lifting property. To show this, it is sufficient to consider those paths in $\beta^{-1}(u)$ of the form $\phi_{t}^{\alpha}(u)$, which is a product of Hamiltonian flows generated by $\alpha^{*} h_{i},\left(h_{i} \in C^{\infty}(P)\right)$. Then $\left(\phi_{t}^{\alpha}(u), x, \phi_{t}^{\rho}(x)\right) \in p_{2}^{-1}(x)$ is clearly a lift of $\phi_{t}^{\alpha}(u)$. It follows immediately from the path lifting property that $p_{1}: p_{2}^{-1}(x) \rightarrow \beta^{-1}(u)$ is a submersion. Furthermore, since $\operatorname{dim} p_{2}^{-1}(x)=\operatorname{dim} \beta^{-1}(u), p_{1}$ is a local diffeomorphism, therefore a covering map. However, $\beta^{-1}(u)$ is simply connected, so $p_{1}$ is a diffeomorphism. Hence, $\Lambda$ is a graph over $\Gamma *_{P} X$.

It is not difficult to see that such a graph $\Lambda$ defines a groupoid $\Gamma$-action on $X$, which can easily be checked to be symplectic. Q.E.D

As in $[\mathrm{X} 1]$, we can introduce the "category" $\zeta(P)$ of complete symplectic realizations for a given Poisson manifold $P$, in which the objects are complete symplectic realizations of $P$, the morphisms from a symplectic realization $X_{1} \stackrel{\rho_{1}}{\rightarrow} P$ to a symplectic realization $X_{2} \stackrel{\rho_{2}}{\rightarrow} P$ are lagrangian submanifolds in $X_{2} *_{P} X_{1}^{-}$, and the composition of morphisms is set-theoretic composition of relations.

Proposition 3.1. Let $(\Gamma \rightrightarrows P, \alpha, \beta)$ be an $\alpha$-simply connected symplectic groupoid, and $X_{1} \stackrel{\rho_{1}}{\rightarrow} P$ and $X_{2} \stackrel{\rho_{2}}{\rightarrow} P$ be complete symplectic realizations of $P$. Then any lagrangian submanifold $\mathscr{L} \subset X_{2} *_{P} X_{1}^{-}$is invariant under the diagonal action of $\Gamma$, where $\Gamma$ acts on $X_{1}$ and $X_{2}$ as defined in Theorem 3.1.

Proof. According to the proof of the preceding theorem, it suffices to show that the flow of the vector field $\left(X_{\rho_{2}^{*} h}, X_{\rho_{1}^{*} h}\right)$ leaves $\mathscr{L}$ invariant for any $h \in C^{\infty}(P)$. Now $\left(X_{\rho_{2}^{*} h}, X_{\rho_{1}^{*} h}\right)$ is a Hamiltonian vector field in $X_{2} \times X_{1}^{-}$generated by the function $\left(\rho_{2}^{*} h\right)\left(x_{2}\right)-\left(\rho_{1}^{*} h\right)\left(x_{1}\right)$, which obviously vanishes on $\mathscr{L}$. Since $\mathscr{L}$ is a lagrangian, $\left(X_{\rho_{2}^{*} h}, X_{\rho_{1}^{*} h}\right)$ is tangent to $\mathscr{L}$. Q.E.D 
Consequently, the "category" of complete symplectic realizations of $P$ coincides with the "category" of symplectic left $\Gamma$-modules.

The following theorem is one of the main results in this section. It plays a key role in proving Theorem 3.3, and is in fact one of the principal motivations behind the introduction of Morita equivalence of Poisson manifolds.

Theorem 3.2. Let $P_{1}$ and $P_{2}$ be integrable Poisson manifolds. $P_{1}$ and $P_{2}$ are Morita equivalent if and only if their $\alpha$-simply connected symplectic groupoids are Morita equivalent.

Proof. Let $\left(G \rightrightarrows P_{1}, \alpha_{1}, \beta_{1}\right)$ and $\left(H \rightrightarrows P_{2}, \alpha_{2}, \beta_{2}\right)$ denote the $\alpha$-simply connected symplectic groupoids of $P_{1}$ and $P_{2}$, respectively.

Suppose that $G$ and $H$ are Morita equivalent with equivalence bimodule $(X ; \rho ; \sigma)$. It follows from Theorem 2.1 in [X1] that $P_{1} \stackrel{\rho}{\leftarrow} X \stackrel{\sigma}{\rightarrow} P_{2}$ is a full dual pair. Each $\rho$-fibre ( $\sigma$-fibre respectively) is diffeomorphic to an $\alpha$-fibre of $H$ (a b-fibre of $G$ respectively), hence is connected and simply connected. Moreover, by Theorem 3.1, both $\rho$ and $\sigma$ are complete Poisson morphisms. Therefore, $P_{1}$ and $P_{2}$ are Morita equivalent as Poisson manifolds.

Conversely, suppose that $P_{1}$ and $P_{2}$ are Morita equivalent integrable Poisson manifolds. According to Theorem 3.1, $X$ becomes a symplectic left $G$-module and a symplectic right $H$-module (note that $\sigma$ is anti-Poisson, so the groupoid action of $H$ should be a right action instead of a left action). We show below that these two actions commute.

Given any $g \in G, x \in X$ and $h \in H$ such that $\beta_{1}(g)=\rho(x)=u$ and $\sigma(x)=\alpha_{2}(h)=v$. We may always assume that $g=\phi^{\alpha_{1}}(u)$ and $h=\psi^{\beta_{2}}(v)$. Then

$$
\begin{aligned}
(g \cdot x) \cdot h & =\psi^{\sigma}(g \cdot x) \\
& =\psi^{\sigma}\left(\phi^{\rho}(x)\right) \\
& =\phi^{\rho}\left(\psi^{\sigma}(x)\right) \\
& =g \cdot(x \cdot h) .
\end{aligned}
$$

It remains to show that the left (right respectively) $G$ ( $H$ respectively)-action on $X$ is free and proper so that $G \backslash X(X / H$ respectively) is anti-Poisson (Poisson respectively) diffeomorphic to $P_{2}\left(P_{1}\right.$ respectively).

To show that $G$ acts on every $\sigma$-fibre freely and transitively, it is sufficient to show that for any $x \in X$, if $u=\rho(x)$ and $v=\sigma(x)$, the map $\lambda: \beta^{-1}(u) \rightarrow \sigma^{-1}(v)$ defined by $\lambda(g)=g \cdot x$ is a diffeomorphism. We shall use the same techniques as in Theorem 3.1. to prove this.

For any $y \in \sigma^{-1}(v)$, since $\sigma^{-1}(v)$ is connected, there exists a product of Hamiltonian flows $\phi^{\rho}$ generated by functions $\rho^{*} h_{i}$ so that $y=\phi^{\rho}(x)$. Take $r=\phi^{\alpha}(u) \in \beta^{-1}(u)$; then $\lambda(r)=r \cdot x=\phi^{\rho}(x)=y$. Thus, $\lambda$ is surjective. In fact, it can be shown exactly by the same argument as above that $\lambda$ has the path lifting property. Therefore, $\lambda$ is a submersion. Moreover, $\operatorname{dim} \sigma^{-1}(v)=\operatorname{dim} \beta^{-1}(u)$ by dimension counting, so $\lambda$ is a local diffeomorphism. Hence, $\lambda$ is a covering map. However, since $\sigma^{-1}(v)$ is simpley connected, $\lambda$ must be a diffeomorphism. Hence, it follows that $\sigma$ induces a diffeomorphism from $G \backslash X$ onto $P_{2}$. This diffeomorphism is anti-Poisson, since $\sigma$ is an anti-Poisson map. Similarly, $\rho$ induces a Poisson 
diffeomorphism from $X / H$ onto $P_{1}$. Therefore, $G$ is Morita equivalent to $H$ as a symplectic groupoid. Q.E.D.

As an immediate consequence of this theorem, we have the following:

Corollary 3.1. The Morita equivalence of Poisson manifolds does give rise to an equivalence relation in the set of all integrable Poisson manifolds.

Another very important consequence of Theorem 3.1, Theorem 3.2 and Proposition 3.1 is the following theorem, which is indeed the main result of this section.

Theorem 3.3. Morita equivalent Poisson manifolds have equivalent "categories" of complete symplectic realizations.

It is of the utmost importance for Morita equivalence of $C^{*}$-algebras that equivalent $C^{*}$-algebras has equivalent categories of representations [Rie1-Rie4]. In some sense, the preceding theorem may be considered as a classical analogue of this fact in Poisson geometry. Indeed, the preceding theorem is our main purpose of introducing Morita equivalence for Poisson manifolds.

Remark. We will see in Sect. 5 that the converse of Theorem 3.3 is not necessarily true as in the case of $C^{*}$-algebras.

\section{Morita Equivalent Regular Poisson Manifolds}

One of the basic questions in the theory of Morita equivalence is the classification of equivalence classes of Morita equivalent Poisson manifolds. This is relatively simple to solve in the case of symplectic manifolds, as we have seen in Sect. 2. However, this question becomes very difficult to answer for general Poisson manifolds. In this section, we attempt to investigate this problem for regular Poisson manifolds. First of all, we need to recall some definitions by Dazord.

Given a regular Poisson manifold $P$ and a symplectic full realization $\rho: X \rightarrow P$, let $\mathscr{F}$ denote the characteristic foliation of $P$ and $\pi: v^{*} \mathscr{F} \rightarrow P$ be the conormal bundle of $\mathscr{F}$. For any $p \in P$ and $\theta \in v_{p}^{*} \mathscr{F}$, we denote $\left(\rho^{*} \theta\right)^{\#}$ as the vector field along $\rho^{-1}(p)$ defined by:

$$
\left.\left(\rho^{*} \theta\right)^{\#}\right\lrcorner \omega=\rho^{*} \theta,
$$

where $\omega$ is the symplectic form on $X$.

Definition 4.1. (Dazord $[D 1, D 2])$ We say that the fibre $\rho^{-1}(p)$ is complete, if for all $\theta \in v_{p}^{*} \mathscr{F}$, the vector field $\left(\rho^{*} \theta\right)^{\#}$ on $\rho^{-1}(p)$ is complete.

Definition 4.2. (Dazord [D1,D2]) A symplectic realization $\rho: X \rightarrow P$ is called a realisation isotrope de Libermann (RIL in abbreviation) if $\rho$ is a surjective submersion and all its fibres are connected, isotropic and complete.

A theorem due to Dazord (Theorem 6.1 [D1]) asserts that any RIL is complete as a Poisson morphism, i.e., a complete symplectic realization. In fact, as an equivalent definition, a RIL is a complete symplectic realization that is a surjective submersion with connected and isotropic fibres.

Suppose that $\left(G \rightrightarrows G_{0}, \alpha_{1}, \beta_{1}\right)$ and $\left(H \rightrightarrows H_{0}, \alpha_{2}, \beta_{2}\right)$ are Morita equivalent symplectic groupoids with equivalence bimodule $(X ; \rho ; \sigma)$. Consider the Stefan 
foliation $\mathscr{F}_{\rho} \cap \mathscr{F}_{\sigma}$ on $X$, where $\mathscr{F}_{\rho}$ and $\mathscr{F}_{\sigma}$ are the foliations on $X$ induced from the $\rho$ and $\sigma$-fibrations, respectively. We have the following:

Proposition 4.1. Suppose that both $G_{0}$ and $H_{0}$ are regular Poisson manifolds.

1. The foliation $\mathscr{F}_{\rho} \cap \mathscr{F}_{\sigma}$ has constant dimension, and its quotient space $X_{1}$ is a Poisson manifold.

2. The natural projection $\tau: X \rightarrow X_{1}$ is a symplectic full realization.

3. The map $\rho_{1}: X_{1} \rightarrow G_{0}\left(\sigma_{1}: X_{1} \rightarrow H_{0}\right.$, respectively) naturally induced from $\rho(\sigma$ respectively) is a Poisson (an anti-Poisson respectively) map.

4. Every symplectic leaf of $X_{1}$ is of the form $\rho_{1}^{-1}\left(L_{G_{0}}\right)=\sigma_{1}^{-1}\left(L_{H_{0}}\right)$, which is symplectically diffeomorphic to $L_{G_{0}} \times L_{H_{0}}^{-}$, where $L_{G_{0}}$ and $L_{H_{0}}^{-}$are related symplectic leaves of $G_{0}$ and $H_{0}$ (see [X1] for the definition of related symplectic leaves), respectively. Therefore, $X_{1}$ is a regular Poisson manifold.

Proof. (1) We first show that the foilation $\mathscr{F}_{\rho} \cap \mathscr{F}_{\sigma}$ coincides with the foilation induced from the $I_{G}$-orbits (or $I_{H}$-orbits) on $X$, where $I_{G}\left(I_{H}\right.$ respectively) is the isotropy groupoid of $G$ ( $H$ respectively).

If $x=g \cdot y$ for some $g \in I_{G}$, then $\sigma(x)=\sigma(g \cdot y)=\sigma(y)$ and $\rho(x)=\rho(g \cdot y)=\alpha_{1}(g)=$ $\beta_{1}(g)=\rho(y)$. In other words, $x$ and $y$ lie in the same leaf of $\mathscr{F}_{\rho} \cap \mathscr{F}_{\sigma}$. Conversely, if $\sigma(x)=\sigma(y)$, then $x=g \cdot y$ for some $g \in G$. Combining this with the condition $\rho(x)=\rho(y)$, we have $\alpha_{1}(g)=\rho(g \cdot y)=\rho(x)=\rho(y)=\beta_{1}(g)$, i.e. $g \in I_{G}$. Similarly, one can prove that the foliation $\mathscr{F}_{\rho} \cap \mathscr{F}_{\sigma}$ coincides with the foliation induced from the $I_{H}$-orbits on $X$. Since $G_{0}$ is a regular Poisson manifold, the isotropy group of the symplectic groupoid $G$ at each point of $G_{0}$ has the same dimension. Hence, the foliation $\mathscr{F}_{\rho} \cap \mathscr{F}_{\sigma}$ has constant dimension. Moreover, since $G$ acts on $X$ freely and properly and $I_{G}$ is a closed subgroupoid of $G, I_{G}$ acts on $X$ freely and properly. Therefore, $X /\left(\mathscr{F}_{\rho} \cap \mathscr{F}_{\sigma}\right)=I_{G} \backslash X$ is a smooth manifold. It is clear that the graph of the foliation $\mathscr{F}_{\rho} \cap \mathscr{F}_{\sigma}: C=\{(x, y) \mid \rho(x)=\rho(y), \sigma(x)=\sigma(y)\}$ is a coisotropic submanifold of $X \times X^{-}$, since the map $\rho \times \sigma: x \mapsto(\rho(x), \sigma(x))$, from $X$ to $G_{0} \times H_{0}^{-}$, is a Poisson map and $C=X *_{(\rho \times \sigma)} X^{-}$. Then it follows immediately that $X_{1}$ naturally becomes a Poisson manifold [W4].

Claims (2) and (3) are quite obvious.

(4) Clearly, $\rho=\rho_{1} \circ \tau$ and $\sigma=\sigma_{1} \circ \tau$. Hence,

$$
\rho_{1}^{-1}\left(L_{G_{0}}\right)=\tau\left(\rho^{-1}\left(L_{G_{0}}\right)\right)=\tau\left(\sigma^{-1}\left(L_{H_{0}}\right)\right)=\sigma_{1}^{-1}\left(L_{H_{0}}\right) .
$$

Since $\rho_{1} \times \sigma_{1}: X_{1} \rightarrow G_{0} \times H_{0}^{-}$is an injective Poisson immersion, $L$ is a symplectic leaf of $X_{1}$ if and only if $\left(\rho_{1} \times \sigma_{1}\right)(L)$ is a symplectic leaf of $G_{0} \times H_{0}^{-}$. Thus, our conclusion follows immediately from this property. Q.E.D.

Remark. It is easy to see that the left $G$-action and the right $H$-action on $X$ induce a left Poisson $G$-action and a right Poisson $H$-action on $X_{1}$, respectively, and these two induced actions commute with each other.

Theorem 4.1. Suppose that $\left(G \rightrightarrows G_{0}, \alpha_{1}, \beta_{1}\right)$ and $\left(H \rightrightarrows H_{0}, \alpha_{2}, \beta_{2}\right)$ are Morita equivalent symplectic groupoids with equivalence bimodule $(X ; \rho ; \sigma)$. If both $G$ and $H$ are $\alpha$-simply connected and their base spaces $G_{0}$ and $H_{0}$ are regular Poisson manifolds, then the morphism $\tau: X \rightarrow X_{1}$, as defined in the preceding proposition, is $a$ RIL. 
Proof. Since every $\tau$-fibre is an intersection of a $\rho$-fibre and a $\sigma$-fibre, it is connected. Moreover, since $\rho$-fibres and $\sigma$-fibres are symplectically orthogonal, all $\tau$-fibres are isotropic. It remains to show that all $\tau$-fibres are complete. By $\mathscr{F}_{G_{0}}$ and $\mathscr{F}_{H_{0}}$, we denote the characteristic foliations of $G_{0}$ and $H_{0}$, respectively, and by $\mathscr{F}$, we denote the characteristic foliation of $X_{1}$. Given any $z \in X_{1}$ and any $\tilde{\omega}$ in the conormal bundle $v_{z}^{*} \mathscr{F}$, there exists an element $\omega$ in the conormal bundle of $\mathscr{F}_{G_{0}}$ at $u=\rho_{1}(z)$, such that $\rho_{1}^{*} \omega=\tilde{\omega}$, since $\mathscr{F}=\rho_{1}^{*} \mathscr{F}_{G_{0}}$. Therefore, $\rho^{*} \omega=\tau^{*} \rho_{1}^{*} \omega=\tau^{*} \tilde{\omega}$. Hence, $\left(\tau^{*} \tilde{\omega}\right)^{\#}=\left(\rho^{*} \omega\right)^{\#}$.

To prove the completeness of $\left(\rho^{*} \omega\right)^{\#}$, let us denote the flow of $\left(\rho^{*} \omega\right)^{\#}$ by $\phi_{t}^{\rho}$ and the flow of $\left(\alpha^{*} \omega\right)^{\#}$ by $\phi_{t}^{\alpha}$. It follows from the $\alpha$-simply connectedness of $G$ that $\phi_{t}^{\rho}(x)=\phi_{t}^{\alpha}(\rho(x)) \cdot x$ for all $x \in X$. Hence $\phi_{t}^{\rho}$ is complete if $\phi_{t}^{\alpha}(\rho(x))$ is a complete flow. However, $\phi_{t}^{\alpha}$ is generated by the vector field $\left(\alpha^{*} \omega\right)^{\#}$, which is tangent to the isotropy group at $\rho(x)$ and is right invariant, in other words, $\left(\alpha^{*} \omega\right)^{\#}$ can be considered as a right invariant vector field of an isotropy group of the groupoid $G$, hence must be complete. Q.E.D.

Theorem 4.2. Suppose that $P_{1}$ and $P_{2}$ are Poisson manifolds with symplectic foliations being the fibrations $\pi_{1}: P_{1} \rightarrow Q_{1}$ and $\pi_{2}: P_{2} \rightarrow Q_{2}$, respectively. $P_{1}$ is Morita equivalent to $P_{2}$ if and only if there exists a diffeomorphism $\psi: Q_{1} \rightarrow Q_{2}$ such that $P_{1} *_{\psi} P_{2}^{-}$has $a \mathrm{RIL}: X \stackrel{\tau}{\rightarrow} P_{1} *_{\psi} P_{2}^{-}$, with $\mathrm{pr}_{1} \circ \tau$ and $\mathrm{pr}_{2} \circ \tau$ having connected and simply connected fibres, where $P_{1} *_{\psi} P_{2}^{-}=\left\{(x, y) \mid x \in P_{1}\right.$ and $y \in P_{2}$ such that $\left.\psi\left(\pi_{1}(x)\right)=\pi_{2}(y)\right\}$ is equipped with the Poisson structure naturally induced from those on $P_{1}$ and $P_{2}^{-}$.

Proof. Let $\rho=p r_{1} \circ \tau$ and $\sigma=p r_{2} \circ \tau$; then both $\rho$ and $\sigma$ are surjective Poisson submersions. For any $f \in C^{\infty}\left(P_{1}\right)$ and $g \in C^{\infty}\left(P_{2}\right)$,

$$
\left\{\rho^{*} f, \sigma^{*} g\right\}=\left\{\tau^{*} \operatorname{pr}_{1}^{*} f, \tau^{*} \operatorname{pr}_{2}^{*} g\right\}=\tau^{*}\left\{\operatorname{pr}_{1}^{*} f, \operatorname{pr}_{2}^{*} g\right\}=0,
$$

so $\rho$-fibres and $\sigma$-fibres are symplectically orthogonal. It follows from dimension counting that $\operatorname{dim} X=\operatorname{dim} P_{1}+\operatorname{dim} P_{2}$. Therefore, the tangent spaces of $\rho$-fibres are the symplectic complements of the tangent spaces of $\sigma$-fibres, and vice versa. According to Theorem 6.1 in [D1], $\tau$ is complete as a Poisson morphism. Since both $\mathrm{pr}_{1}$ and $\mathrm{pr}_{2}$ are complete Poisson morphisms by construction, $\rho$ and $\sigma$ are complete. Therefore, $P_{1}$ and $P_{2}$ are Morita equivalent as Poisson manifolds.

Conversely, if $P_{1}$ and $P_{2}$ are Morita equivalent, there exists an equivalence bimodule $X: P_{1} \stackrel{\rho}{\leftarrow} X \stackrel{\sigma}{\rightarrow} P_{2}$. Let $G$ and $H$ be the $\alpha$-simply connected symplectic groupoids over $P_{1}$ and $P_{2}$, respectively. Then $G$ and $H$ are Morita equivalent as symplectic groupoids. Intoduce a map $\psi$ from $Q_{1}$ to $Q_{2}$ as follows. For any $x \in Q_{1}$, corresponding to the symplectic leaf $\pi_{1}^{-1}(x)$ in $P_{1}$, there is a unique symplectic leaf of $P_{2}$ assumed to be $\pi_{2}^{-1}(y)$ for some $y \in Q_{2}$. Then, this correspondence $x \mapsto y$ defines a map $\psi$ from $Q_{1}$ to $Q_{2}$. It is clear that this map $\psi$ is in fact a diffeomorphism from $Q_{1}$ onto $Q_{2}$ and $P_{1} *_{\psi} P_{2}^{-}$is Poisson diffeomorphic to $X_{1}$, the Poisson manifold introduced in Proposition 4.1. Hence, it follows from Theorem 4.2 that $\tau: X \rightarrow P_{1} *_{\psi} P_{2}^{-}\left(\cong X_{1}\right)$ is a RIL. Q.E.D.

As a direct consequence of this theorem, we have the following condition characterizing regular Poisson manifolds Morita equivalent to zero Poisson manifolds. 
Theorem 4.3. Let $P$ be a regular Poisson manifold with symplectic fibration $\pi: P \rightarrow Q$ and $[\zeta]$ the fundamental class of $P . P$ is Morita equivalent to $Q$ with zero Poisson structure if and only if all the symplectic leaves of $P$ are simply connected and $[\zeta]=0$.

Proof. Suppose that $P$ is Morita equivalent to $Q$ equipped with the zero Poisson structure. According to Theorem 4.2, there is a diffeomorphism $\psi: Q \rightarrow Q$ such that $P *_{\psi} Q^{-}$has a RIL. Without loss of generality, we may always assume that $\psi=\mathrm{id}$ so that $P *_{\psi} Q^{-}=P$. Therefore, we have a RIL $\tau: X \rightarrow P$ such that both $\tau$ and $\pi^{\circ} \tau$ have connected and simply connected fibres. By $\mathscr{R}$, we denote the réseau over $P$ associated to this $\mathrm{RIL}$; then $\tau^{-1}(p) \cong v_{p}^{*} \mathscr{F} / \mathscr{R}_{p}$ for any $p \in P$, where $\mathscr{F}$ is the characteristic foliation of $P$ and $v^{*} \mathscr{F}$ is the conormal bundle of $\mathscr{F}$ (see [D1, D2] for more details). Since both $\tau^{-1}(p)$ and $v_{p}^{*} \mathscr{F}$ are simply connected and $\mathscr{R}_{p}$ is a discrete group, $\mathscr{R}_{p}$ must be 0 . Hence, $\mathscr{R}=0$. It follows from the equation $d_{\mathscr{R}}^{*} v=[\zeta]$ (Theorem $5.2[\mathrm{D} 2]$ ) that $[\zeta]=0$.

Moreover, for any $q \in Q$, from the fibration $(\tau$-fibre $) \rightarrow(\pi \circ \tau)^{-1}(q) \rightarrow \pi^{-1}(q)$, it follows that

$$
0=\pi_{1}\left((\pi \circ \tau)^{-1}(q)\right) \rightarrow \pi_{1}\left(\pi^{-1}(q)\right) \rightarrow \pi_{0}(\tau \text {-fibre })=0 .
$$

Thus, $\pi_{1}\left(\pi^{-1}(q)\right)=0$. In other words, every symplectic leaf of $P$ is simply connected.

Conversely, if $[\zeta]=0$, then $\tau: v^{*} \mathscr{F} \rightarrow P$ with the symplectic form $\tau^{*} \tilde{\sigma}+i^{*} d \lambda$ is a RIL of $P$ (cf. [D1]), where $\tilde{\sigma} \in \Omega^{2}\left(T^{*} P\right.$ ) is a closed extension of the symplectic forms along symplectic leaves of $P, d \lambda$ is the standard symplectic structure on $T^{*} P$ and $i: v^{*} \mathscr{F} \rightarrow T^{*} P$ is the natural inclusion. It is quite obvious that $\tau^{-1}(p)=v_{p}^{*} \mathscr{F}$ is simply connected for any $p \in P$. Given any symplectic leaf $L$ of $P$, which is assumed to be simply connected, it follows from the exact sequence

$$
0=\pi_{1}(\tau \text {-fibre }) \rightarrow \pi_{1}\left(\tau^{-1}(L)\right) \rightarrow \pi_{1}(L)=0
$$

associated to the fibration: $(\tau$-fibre $) \rightarrow \tau^{-1}(L) \rightarrow L$ that $\tau^{-1}(L)$ is simply connected. The conclusion thus follows immediately from Theorem 4.1. Q.E.D.

Remark. It would be interesting to figure out the relation between the fundamental class of Poisson manifolds and the Dixmier-Douady invariant of continuous trace $C^{*}$-algebras [Dix]. It seems that the preceding theorem is just a classical analogue of a well-known similar fact in $C^{*}$-algebras.

\section{Morita Equivalence Invariants}

In this section, we will continue to study Morita equivalence of regular Poisson manifolds. In particular, we will concentrate on the simplest case of Poisson manifolds where the characteristic foliations are trivial fibrations $P=S \times Q \rightarrow Q$. In this case, a complete invariant of Morita equivalence is obtained, which we hope is still valid for general Poisson manifolds with locally trivial symplectic fibrations. First of all, we start with a purely topological result.

Theorem 5.1. Let $T^{k} \rightarrow E \rightarrow S$ be a torus principal bundle with Chern class $\left(\left[C_{1}\right], \ldots,\left[C_{k}\right]\right) . E$ is simply connected if and only if $S$ is simply connected and $\operatorname{per}\left(\left[C_{1}\right], \ldots,\left[C_{k}\right]\right)=\mathbf{Z}^{k}$, where $\operatorname{per}\left(\left[C_{1}\right], \ldots,\left[C_{k}\right]\right)$ is the image of the group homomorphism $\varphi: H_{2}(S, Z) \rightarrow \mathbf{Z}^{k}$ defined by $\varphi(c)=\left(\left\langle\left[C_{1}\right], c\right\rangle, \ldots,\left\langle\left[C_{k}\right], c\right\rangle\right)$ for all $c \in \mathrm{H}_{2}(S, \mathbf{Z})$. 
Proof. From the exact sequence

$$
\pi_{2}(S) \stackrel{\phi}{\rightarrow} \pi_{1}\left(T^{k}\right) \rightarrow \pi_{1}(E) \rightarrow \pi_{1}(S) \rightarrow \pi_{0}\left(T^{k}\right)=0,
$$

it follows that if $E$ is simply connected, so is $S$. Hence, it suffices to show that the statement $E$ is simply connected is equivalent to $\operatorname{per}\left(\left[C_{1}\right], \ldots,\left[C_{k}\right]\right)=\mathbf{Z}^{k}$ under the simply connectedness assumption on $S$. First, it follows from the universal coefficients theorem that $\mathrm{H}^{2}(S, \mathbf{Z})=\operatorname{Hom}\left(\mathrm{H}_{2}(S, \mathbf{Z}), \mathbf{Z}\right)$ if $S$ is simply connected.

Let $T^{k} \rightarrow E T^{k} \rightarrow B T^{k}$ be the universal bundle. There exists a map $f: S \rightarrow B T^{k}$ such that $E=f^{*}\left(E T^{k}\right)$. Then $f^{*}: H^{2}\left(B T^{k}, \mathbf{Z}\right) \rightarrow H^{2}(S, \mathbf{Z})$ maps a family of generators of $H^{2}\left(B T^{k}, \mathbf{Z}\right)$ to the Chern class $\left(\left[C_{1}\right], \ldots,\left[C_{k}\right]\right)$. In other words, if we identify $H^{2}\left(B T^{k}, \mathbf{Z}\right)$ with $\mathbf{Z}^{k}$, then $f^{*} \operatorname{maps}\left(n_{1}, \ldots, n_{k}\right)$ to $n_{1}\left[C_{1}\right]+\cdots+n_{k}\left[C_{k}\right]$. Hence, $f_{*}: H_{2}(S, \mathbf{Z}) \rightarrow H_{2}\left(B T^{k}, \mathbf{Z}\right)=\mathbf{Z}^{k}$ is given by $c \rightarrow\left(\left\langle C_{1}, c\right\rangle, \ldots,\left\langle C_{k}, c\right\rangle\right)$. Therefore, $f_{*}\left(H_{2}(S, Z)\right)=\operatorname{per}\left(\left[C_{1}\right], \ldots,\left[C_{k}\right]\right)$.

On the other hand, the homomorphism $\phi: \pi_{2}(S) \rightarrow \pi_{1}\left(T^{k}\right)$ in the exact sequence (2) is the composition of $f_{*}: \pi_{2}(S)\left(=H_{2}(S, \mathbf{Z})\right) \rightarrow \pi_{2}\left(B T^{k}\right)\left(=H_{2}\left(B T^{k}, \mathbf{Z}\right)\right)$ with $\delta: \pi_{2}\left(B T^{k}\right) \rightarrow \pi_{1}\left(T^{k}\right)$. Hence, saying that $E$ is simply connected is equivalent to saying that $\phi$ is surjective, or that $f_{*}$ is surjective. In other words, it is equivalent to $\operatorname{per}\left(\left[C_{1}\right], \ldots,\left[C_{k}\right]\right)=\mathbf{Z}^{k}$. Q.E.D.

Given a Poisson manifold in which the characteristic foliation is a trivial fibration $\pi: P=S \times Q \rightarrow Q$, the Poisson structure is described by a smooth map from $Q$ to $Z^{2}(S)$, the space of all closed two-forms on $S$, denoted by $\left\{\omega_{y} \mid y \in Q\right\}$.

A theorem due to Dazord [D1] asserts that $P$ is integrable if and only if

$$
\left[\omega_{y}\right]=\left[\omega_{0}\right]+\sum_{i=1}^{k} g_{i}(y)\left[C_{i}\right], \quad \forall y \in Q
$$

for some $g_{1}(y), \ldots, g_{k}(y) \in C^{\infty}(Q)$ and $\left[C_{1}\right], \ldots,\left[C_{k}\right] \in H^{2}(S, \mathbf{Z})$ such that $\left(g_{1}, \ldots, g_{k}\right)$ : $Q \rightarrow \mathbf{R}^{k}$ is a submersion and $\left[C_{1}\right], \ldots,\left[C_{k}\right]$ are linearly independent in $H^{2}(S, \mathbf{R})$.

Note that $\operatorname{per}\left(\left[C_{1}\right], \ldots,\left[C_{k}\right]\right)$ is then a subgroup of $\mathbf{Z}^{k}$ of rank $k$. In general, $\operatorname{per}\left(\left[C_{1}\right], \ldots,\left[C_{k}\right]\right) \neq \mathbf{Z}^{k}$. However, we can always choose $\left\{g_{i}\right\}$ and $\left\{\left[C_{i}\right]\right\}$ suitably so that $\operatorname{per}\left(\left[C_{1}\right], \ldots,\left[C_{k}\right]\right)=\mathbf{Z}^{k}$. Below, we say $\left\{\omega_{y}\right\}$ is of a standard form if it is given by Eq. (3) and $\operatorname{per}\left(\left[C_{1}\right], \ldots,\left[C_{k}\right]\right)=\mathbf{Z}^{k}$.

In fact, any Poisson manifold with the Poisson structure given by Eq. (3) admits a RIL [D1, D2], which can be constructed as follows. First, note that there is a family of one-forms $\left\{\theta_{y} \mid y \in Q\right\}$ on $S$ such that

$$
\omega_{y}=\omega_{0}+\sum_{i=1}^{k} g_{i}(y) C_{i}+d_{S} \theta_{y},
$$

where $d_{S}$ is the exterior differential with respect to $S$. Let $E \stackrel{p}{\rightarrow} S$ be a $T^{k}$ principal bundle with connection $\left(\theta_{1}, \ldots, \theta_{k}\right)$ and curvature $\left(C_{1}, \ldots, C_{k}\right)$. Let

$$
\begin{aligned}
& \Gamma=E \times\left(T^{*} Q / \sum_{i=1}^{k} \mathbf{R} d g_{i}\right) \text { and } \\
& \omega=p^{*}\left(\omega_{0}+\sum_{i=1}^{k} g_{i}(y) C_{i}\right)+\phi^{*}\left(d \theta_{y}\right)+\sum_{i=1}^{k} \pi^{*}\left(d g_{i}(y)\right) \wedge \theta_{i}+\left(d \lambda+\sum_{i=1}^{k} d g_{i} \wedge \gamma_{i}\right),
\end{aligned}
$$



where $\theta_{y}$ is considered as a one-form on $P$ of type $(1,0),\left(T^{*} Q / \sum_{i=1}^{k} \mathbf{R} d g_{i}\right)$ is the
quotient space of $T^{*} Q$ under the $\mathbf{R}^{k}$-action defined by

$$
\left(t_{1}, \ldots, t_{k}\right)(y, \xi)=\left(y, \xi+\sum_{i=1}^{k} t_{i} d g_{i}(y)\right), \quad \forall(y, \xi) \in T^{*} Q
$$

$\pi:\left(T^{*} Q / \sum_{i=1}^{k} \mathbf{R} d g_{i}\right) \rightarrow Q$ is the natural projection, $\left(\gamma_{1}, \ldots, \gamma_{k}\right)$ is a connection of this principal bundle, $d \lambda$ is the standard symplectic 2 -form on $T^{*} Q$ and $\phi=p \times \pi$. Then $(\Gamma, \omega) \stackrel{\phi}{\rightarrow} P$ is a RIL. Now we can state our main theorem of this section as the following:

Theorem 5.2. Let $P_{1}=S_{1} \times Q_{1}$ and $P_{2}=S_{2} \times Q_{2}$ be Poisson manifolds with Poisson structures $\left\{\omega_{y} \mid y \in Q_{1}\right\}$ and $\left\{\sigma_{z} \mid z \in Q_{2}\right\}$, respectively. Suppose that both $S_{1}$ and $S_{2}$ are simply connected. Then $P_{1}$ is Morita equivalent to $P_{2}$ if and only if $\left\{\omega_{y}\right\}$ and $\left\{\sigma_{z}\right\}$ have the following standard forms:

$$
\begin{aligned}
& {\left[\omega_{y}\right]=\left[\omega_{0}\right]+\sum_{i=1}^{k} g_{i}(\psi(y)) C_{i},} \\
& {\left[\sigma_{z}\right]=\left[\sigma_{0}\right]+\sum_{i=1}^{k} g_{i}(z) D_{i}}
\end{aligned}
$$

for some diffeomorphism $\psi: Q_{1} \rightarrow Q_{2}$.

Proof. Suppose that $P_{1}$ and $P_{2}$ are Morita equivalent. By Theorem 4.2, there exists a diffeomorphism $\psi: Q_{1} \rightarrow Q_{2}$ such that $P_{1} *_{\psi} P_{2}^{-}$has a RIL. Under this diffeomorphism $\psi$, we may assume that $Q_{1}=Q_{2}=Q$. Then, $P_{1} *_{\psi} P_{2}^{-}$is Poisson diffeomorphic to $S_{1} \times S_{2}^{-} \times Q$ with Poisson structure given by $\left\{\left(\omega_{y},-\sigma_{y}\right) \mid y \in Q\right\}$. Since $P_{1} *_{\psi} P_{2}^{-}$admits a RIL, according to Dazord [D1, D2], $\left\{\left(\omega_{y},-\sigma_{y}\right)\right\}$ has a standard form:

$$
\left[\left(\omega_{y},-\sigma_{y}\right)\right]=\left[\left(\omega_{0}, \sigma_{0}\right)\right]+\sum_{i=1}^{k} g_{i}(y)\left[\left(C_{i},-D_{i}\right)\right]
$$

Therefore,

and

$$
\left[\omega_{y}\right]=\left[\omega_{0}\right]+\sum_{i=1}^{k} g_{i}(y)\left[C_{i}\right]
$$

$$
\left[\sigma_{y}\right]=\left[\sigma_{0}\right]+\sum_{i=1}^{k} g_{i}(y)\left[D_{i}\right]
$$

It remains for us to show that $\operatorname{per}\left(\left[C_{1}\right], \ldots,\left[C_{k}\right]\right)=\operatorname{per}\left(\left[D_{1}\right], \ldots,\left[D_{k}\right]\right)=\mathbf{Z}^{k}$. Let $\tau: X \rightarrow S_{1} \times S_{2}^{-} \times Q$ be a RIL, such that $\operatorname{pr}_{1} \circ \tau$ and $\operatorname{pr}_{2} \circ \tau$ have connected and simply connected fibres, and let $\mathscr{R}$ be the réseau of this RIL. We may always write $\mathscr{R}=p^{*} \mathscr{R}^{Q}$ for a lattice $\mathscr{R}^{Q}$ over $Q$, where $p$ is the natural projection from $S_{1} \times S_{2}^{-} \times Q$ onto $Q$ [D1, D2]. By Theorem 6.1 in [D2], $\tau$ induces a RILC:

$$
\tau^{\prime}: X \rightarrow\left(S_{1} \times S_{2}^{-} \times T^{*} Q\right) / \mathscr{R} \otimes \mathbf{R} \cong S_{1} \times S_{2}^{-} \times\left(T^{*} Q / \mathscr{R}^{Q} \otimes \mathbf{R}\right),
$$

which is in fact a $T^{k}$-principal bundle having Chern class $\left(\left[C_{1},-D_{1}, 0\right], \ldots\right.$, 
$\left.\left[C_{k},-D_{k}, 0\right]\right)$ under a suitable basis. Let $X_{s, y}=\tau^{-1}\left(S_{1} \times\{s\} \times\{y\}\right)$ for some fixed $s \in S_{2}$ and $y \in Q$. Then $X_{s, y}=\left(\operatorname{pr}_{2}{ }^{\circ} \tau\right)^{-1}(s, y)$ is simply connected. On the other hand, $\tau^{\prime}: X_{s, y} \rightarrow S_{1} \times\{s\} \times\left(T_{y}^{*} Q / \mathscr{R}_{y}^{Q} \otimes \mathbf{R}\right)$ is clearly a $T^{k}$-principal bundle with Chern class $\left(\left[C_{1}, 0\right], \ldots,\left[C_{k}, 0\right]\right)$. It follows immediately from Theorem 5.1 that $\operatorname{per}\left(\left[C_{1}\right], \ldots,\left[C_{k}\right]\right)=$ $\mathbf{Z}^{k}$. Similarly, per $\left(\left[D_{1}\right], \ldots,\left[D_{k}\right]\right)=\mathbf{Z}^{k}$.

Conversely, without loss of generality, we assume that $Q_{1}=Q_{2}=Q$, and $P_{1}=S_{1} \times Q$ and $P_{2}=S_{2} \times Q$ have Poisson structures $\left\{\omega_{y}\right\}$ and $\left\{\sigma_{y}\right\}$ given by the standard forms:

$$
\left[\omega_{y}\right]=\left[\omega_{0}\right]+\sum_{i=1}^{k} g_{i}(y)\left[C_{i}\right]
$$

and

$$
\left[\sigma_{y}\right]=\left[\sigma_{0}\right]+\sum_{i=1}^{k} g_{i}(y)\left[D_{i}\right],
$$

respectively. Thus $P=P_{1} *_{\psi} P_{2}^{-}=S_{1} \times S_{2}^{-} \times Q$ has Poisson structure $\left\{\left(\omega_{y},-\sigma_{y}\right)\right\}$. According to the observation preceding Theorem 5.2, $P$ has a RIL of the form $\phi: E \times T^{*} Q /\left(\sum_{i=1}^{k} \mathbf{R} d g_{i}\right) \rightarrow P$, where $E$ can be taken to be any $T^{k}$-principal bundle over $S_{1} \times S_{2}^{-}$with Chern class $\left(\left[C_{1},-D_{1}\right], \ldots,\left[C_{k},-D_{k}\right]\right)$. In fact, we can construct $E$ in the following manner. Take a $T^{k}$-principal bundle $E_{1}$ over $S_{1}$ with Chern class $\left(\left[C_{1}\right], \ldots,\left[C_{k}\right]\right)$ and a $T^{k}$-principal bundle $E_{2}$ over $S_{2}$ with Chern class $\left(\left[-D_{1}\right], \ldots,\left[-D_{k}\right]\right)$. Let $E=\left(E_{1} \times E_{2}\right) / T^{k}$, with $T^{k}$ acting on $E_{1} \times E_{2}$ diagonally. It follows from Theorem 5.1 that both $E_{1}$ and $E_{2}$ are simply connected. By $\pi_{i}$, we denote the natural projection from $P$ onto $P_{i},(i=1,2)$. Then for any $(s, y) \in P_{1}=S_{1} \times Q$,

$$
\left(\pi_{1}^{\circ} \circ\right)^{-1}(s, y)=\phi^{-1}\left(\{s\} \times S_{2} \times\{y\}\right) \cong E_{2} \times\left(T_{y}^{*} Q / \sum_{i=1}^{k} \mathbf{R} d g_{i}(y)\right),
$$

which is obviously connected and simply connected. Similarly, every $\left(\pi_{2}^{\circ} \phi\right)$-fibre is also connected and simply connected. Q.E.D.

Remark. With the aid of the preceding theorem, we can construct a counterexample to the converse of Theorem 3.3. Take a manifold $Q$ and a function $g \in C^{\infty}(Q)$ such that $d g \neq 0$ everywhere and $g \neq 2 g \circ \psi$ for all diffeomorphisms $\psi: Q \rightarrow Q$, which is always possible. For instance, we can take $Q=(0,1)$ and $g(y)=y$. Let $S^{2}$ be the two-sphere with the standard symplectic structure $\omega$, and let $P_{1}=S^{2} \times Q$ with the Poisson structure $\omega_{y}=g(y) \omega$ and $P_{2}=S^{2} \times Q$ with the Poisson structure $\omega_{y}=2 g(y) \omega$. Then, according to Theorem 5.2, $P_{1}$ and $P_{2}$ are not Morita equivalent. However, it is obvious that $P_{1}$ and $P_{2}$ have equivalent "categories" of complete symplectic realizations.

Note that the standard form in Eq. (3) is not unique. In fact, if both $\left[\omega_{y}\right]=$ $\left[\omega_{0}\right]+\sum_{i=1}^{k} g_{i}(y)\left[C_{i}\right]$ and $\left[\omega_{y}^{\prime}\right]=\left[\omega_{0}^{\prime}\right]+\sum_{i=1}^{k} g_{i}(y)\left[C_{i}^{\prime}\right]$ are standard forms of $\left\{\omega_{y}\right\}$, then $\left(\left[C_{1}\right], \ldots,\left[C_{k}\right]\right)$ and $\left(\left[C_{1}^{\prime}\right], \ldots,\left[C_{k}^{\prime}\right]\right)$ differ by a linear transformation of $G l(k, \mathbf{Z})$, as do $\left(d g_{1}, \ldots, d g_{k}\right)$ and $\left(d g_{1}^{\prime}, \ldots, d g_{k}^{\prime}\right)$. In order to find out the intrinsic invariants of Morita equivalence, we need to recall the construction of the variation lattices $\mathscr{R}^{Q}$, first introduced by Dazord [D1,D2]. It will be useful later on to look 
at this construction in a slightly more general context. Let $P$ be a Poisson manifold with locally trivial symplectic fibration $\pi: P \rightarrow Q$. Associated to this fibration, there is a vector bundle $H^{2}(S, \mathbf{R}) \rightarrow \Xi^{2} \rightarrow Q$, which has a natural flat connection $\nabla$. Here $S$ is the symplectic leaf of $P$. The Poisson structure on $P$ defines a global section of this bundle, which is denoted by $\omega$. Define a discrete subbundle $\mathscr{R}^{Q}$ of $T^{*} Q$ as follows. For any $y \in Q, \xi \in \mathscr{R}_{y}^{Q} \subset T_{y}^{*} Q$ if and only if there is a $c \in H_{2}\left(\pi^{-1}(y), \mathbf{Z}\right)$ such that

$$
\langle\xi, v\rangle=\int_{c} \nabla_{v} \omega, \quad \forall v \in T_{y} Q .
$$

$\mathscr{R}^{Q}$ is a covering space over $Q$, which plays a crucial role in the Poisson integrability theory of Dazord (see [D1, D2] for more details). Since $\mathscr{R}^{Q}$ measures the variations of symplectic structures along symplectic leaves of the Poisson manifold $P$, we call it the variation lattice of $P$.

In particular, if $P=S \times Q$ with Poisson structure $\left\{\omega_{y}\right\}$ given by a standard form as in Eq. (3), then $\mathscr{R}^{Q}=\sum_{i=1}^{k} \mathbf{Z} d g_{i}$. Conversely, we have

Lemma 5.1. If the variation lattice $\mathscr{R}^{Q}$ of a Poisson manifold $P=S \times Q$ is $\sum_{i=1}^{k} \mathbf{Z} d g_{i}$ and $S$ is simply connected, then $\left\{\omega_{y}\right\}$ has a standard form given by Eq. (3).

This fact is more or less contained in [D2]. Here, for completeness, we outline a proof.

Proof. By $\Phi_{y}$, we denote the group homomorphism from $H_{2}(S, \mathbf{Z})$ to $\sum_{i=1}^{k} \mathbf{Z} d g_{i}(y) \subset T_{y}^{*} Q$ given by $\Phi_{y}(c)=\int_{c} d \omega_{y}, \forall c \in H_{2}(S, \mathbf{Z})$. Fix some point $y_{0} \in Q$, and let $\Phi_{0}=\Phi_{y_{0}}$. Then, there are $c_{1}, \ldots, c_{k} \in H_{2}(S, \mathbf{Z})$ such that $\Phi_{0}\left(c_{i}\right)=d g_{i}\left(y_{0}\right)$, $(i=1, \ldots, k)$. Obviously, $c_{1}, \ldots, c_{k}$ are linearly independent in $H_{2}(S, \mathbf{R})$, since $d g_{1}\left(y_{0}\right), \ldots, d g_{k}\left(y_{0}\right)$ are linearly independent. Now, clearly we have the decomposition

$$
H_{2}(S, \mathbf{Z})=\operatorname{ker} \Phi_{0} \oplus \operatorname{span}\left\{c_{1}, \ldots, c_{k}\right\} .
$$

Since $H^{2}(S, \mathbf{Z})=\operatorname{Hom}\left(H_{2}(S, \mathbf{Z}), \mathbf{Z}\right)$, there exist $\left[C_{1}\right], \ldots,\left[C_{k}\right] \in H^{2}(S, \mathbf{Z})$ so that $\left\langle\left[C_{i}\right], c_{j}\right\rangle=\delta_{i j}$ and $\left\langle\left[C_{i}\right], \operatorname{ker} \Phi_{0}\right\rangle=0, \forall i, j$. Thus, it is clear that $\operatorname{per}\left(\left[C_{1}\right], \ldots,\left[C_{k}\right]\right)=$ $\mathbf{Z}^{k}$ and

$$
\Phi_{0}(c)=\sum_{i=1}^{k} \Phi_{0}\left(c_{i}\right)\left\langle\left[C_{i}\right], c\right\rangle, \quad \forall c \in H_{2}(S, \mathbf{Z})
$$

Since $\sum_{i=1}^{k} \mathbf{Z} d g_{i}$ is a discrete bundle, $\Phi_{y}\left(c_{i}\right)=d g_{i}(y)$ and $\operatorname{ker} \Phi_{y}=\operatorname{ker} \Phi_{0}$ for all $y \in Q$ and $i=1, \ldots, k$. Hence, $\Phi_{y}(c)=\sum_{i=1}^{k} \Phi_{y}\left(c_{i}\right)\left\langle\left[C_{i}\right], c\right\rangle, \forall c \in H_{2}(S, \mathbf{Z})$ and $y \in Q$, i.e.,

$$
\int_{c} d \omega_{y}=\int_{c} \sum_{i=1}^{k} d g_{i}(y)\left[C_{i}\right]
$$

Therefore, $d\left[\omega_{y}\right]=\sum_{i=1}^{k} d g_{i}(y)\left[C_{i}\right]$. Thus, the conclusion follows immediately. 
It is worth noting that $\sum_{i=1}^{k} \mathbf{Z} d g_{i}=\sum_{i=1}^{k} \mathbf{Z} d h_{i}$ if and only if $\left(d g_{1}, \ldots, d g_{k}\right)$ and $\left(d h_{1}, \ldots, d h_{k}\right)$ differ by a linear transformation of $G l(k ; \mathbf{Z})$. Finally, with Lemma 5.1, we can formulate the following equivalent version of Theorem 5.2.

Theorem 5.3. Let $P_{1}$ and $P_{2}$ be Poisson manifolds as in Theorem 5.2, and let $\mathscr{R}_{1}^{Q}$ and $\mathscr{R}_{2}^{Q}$ be their variation lattices, respectively. $P_{1}$ and $P_{2}$ are Morita equivalent if and only if there is diffeomorphism $\psi: Q_{1} \rightarrow Q_{2}$ such that $\psi^{*}\left(\mathscr{R}_{2}^{Q}\right)=\mathscr{R}_{1}^{Q}$.

We conclude this section by the following:

Conjecture. Suppose that $P_{1}$ and $P_{2}$ are Poisson manifolds with symplectic fibrations $\pi_{1}: P_{1} \rightarrow Q_{1}$ and $\pi_{2}: P_{2} \rightarrow Q_{2}$, respectively, so that both $\pi_{1}$ and $\pi_{2}$ are locally trivial as fibrations and have connected and simply connected fibres. Let $\mathscr{R}_{1}^{Q}$ and $\mathscr{R}_{2}^{Q}$ be the variation lattices of $P_{1}$ and $P_{2}$, respectively. $P_{1}$ and $P_{2}$ are Morita equivalent if and only if there is a diffeomorphism $\psi: Q_{1} \rightarrow Q_{2}$ such that $\psi^{*}\left(\mathscr{R}_{2}^{Q}\right)=\mathscr{R}_{1}^{Q}$.

Acknowledgements. This is a part of the author's Ph.D thesis in Mathematics at the University of California, Berkeley. The author owes a great debt of gratitude to his thesis adviser, Professor Alan Weinstein, whose knowledge and insight have contributed tremendously to this work. He also wants to acknowledge his great indebtedness of Professor Marc Rieffel, another member of his dissertation committee, for his invaluable guidance and continual support during the preparation of this work. Finally, he wishes to thank Professor Pierre Dazord, Professor Richard Lashof, Professor Kirill Mackenzie and Dr. Jianghua Lu for many stimulating discussions, and the referee for pointing out an error in the manuscript.

\section{References}

[B] Breen, L.: Bitoreseurs et cohomologie non abélienne. The Grothendieck Festschrift, vol. I, 401-476 (1990)

[CDW] Coste, A., Dazord, P., Weinstein, A.: Groupoïdes symplectiques. Publications du Départment de Mathématique, Université Claude Bernard Lyon I (1987)

[D1] Dazord, P.: Groupoïdes symplectiques et troisième théorème de Lie "non linéaire." Lecture Notes in Mathematics, vol. 1416, pp. 39-74. Berlin, Heidelberg, New York: Springer 1990

[D2] Dazord, P.: Réalisations isotropes de Libermann. Publ. Dept. Math. Lyon (1989)

[Dix] Dixmier, J.: $C^{*}$-algebras. New York: North Holland 1977

[Ka] Karasev, M. V.: Analogues of objects of the theory of Lie groups for nonlinear Poisson brackets. Math. USSR Izv. 28, 497-527 (1987)

[L] Lichnerowicz, A.: Les variétés de Poisson et leurs algèbres de Lie associées. J. Diff. Geom. 12, 253-300 (1977)

[M1] Mackenzie, K.: Lie groupoids and Lie algebroids in differential geometry. LMS Lecture Notes Series, vol. 124. Cambridge University Press 1987

[Mo] Morita, K.: Duality for modules and its applications to the theory of rings with minimum condition. Tokyo Kyoiku Diagaku 6, 83-142 (1958)

[MiW] Mikami, K., Weinstein, A.: Moments and reduction for symplectic groupoid actions. Publ. RIMS Kyoto Univ. 24, 121-140 (1988)

[MRW] Muhly, P., Renault, J., Williams, D.: Equivalence and isomorphism for groupoid $C^{*}$-algebras. J. Operator Theory 17, 3-22 (1987)

[Rie1] Rieffel, M. A.: Induced representations of $C^{*}$-algebras. Adv. Math. 13, 176-257(1974)

[Rie2] Rieffel, M. A.: Morita equivalence for operator algebras. Proc. Symp. Pure Math. 38, 285-298 (1982) 
[Rie3] Rieffel, M. A.: Applications of strong Morita equivalence to transformation group $C^{*}$-algebras. Proc. Symp. Pure Math. 38, 299-310 (1982)

[Rie4] Rieffel, M. A.: Morita equivalence for $C^{*}$-algebras and $W^{*}$-algebras. J. Pure Appl. Algebra 5, 51-96 (1974)

[W0] Weinstein, A.: The symplectic "cateogory." Lecture Notes in Mathematics, vol. 905, pp. 45-50. Berlin, Heidelberg, New York: Springer 1982

[W1] Weinstein, A.: The local structure of Poisson manifolds. J. Diff. Geom. 18, 523-557(1983)

[W2] Weinstein, A.: Symplectic groupoids and Poisson manifolds. Bull. Am. Math. Soc. 16, 101-104 (1987)

[W3] Weinstein, A.: Affine Poisson structures. Int. J. Math. 1, 343-360 (1990)

[W4] Weinstein, A.: Coisotropic calculus and Poisson groupoids. J. Math. Soc. Jpn. 40, 705-727 (1988)

[WX] Weinstein, A., Xu, P.: Extensions of symplectic groupoids and quantization. J. Reine Angew. Math. 417, 159-189 (1991)

[X1] Xu, P.: Morita equivalent symplectic groupoids, Seminaire Sud-Rhodanien à Berkeley, Symplectic geometry, groupoids, and integrable systems. Springer-MSRI publications, pp. 291-311 (1991)

[X2] Xu, P.: Symplectic realizations of Poisson manifolds. Ann. Scient. Éc. Norm. Sup. (to appear)

Communicated by A. Connes 
